\section{Optimized Blood Volume Monitoring during Hemodialysis Procedure based on Ultrasonic Speed Measurement}

\author{
Nafisi V. R. ${ }^{* *(0)}$, Eghbal M. ${ }^{2}$
}

\begin{abstract}
Fast reduction of blood water volume due to ultra-filtration via hemodialysis machine and critical threshold of blood water volume can result in clinical complications such as hypotension. When blood water volume reduces during hemodialysis process, the concentration of blood forming elements will increase. One of the methods for the monitoring of the concentration changes is ultrasonic speed measurement.

In this paper, we optimize the ultrasonic speed measurement method in such a way that it would be compatible with conventional air detector in a hemodialysis machine. Air bubble detecting and the relative blood water volume estimating can be achieved by adding the "sing-around" ultrasonic speed measurement method to the current hemodialysis machine; air bubble detection is achieved by monitoring the decline in the ultrasonic wave amplitude (because of air bubbles) and the protein concentration measurement is achieved by measuring the transit time of the wave. Therefore, the optimized method can be applied to the air detection device of a hemodialysis machine.

Citation: Nafisi V. R, Eghbal M. Optimized Blood Volume Monitoring during Hemodialysis Procedure based on Ultrasonic Speed Measurement. J Biomed Phys Eng. 2019;9(3):373-380. https://doi.org/10.31661/jbpe.v9i3Jun.675.
\end{abstract}

\section{Keywords}

Hemodialysis, Blood Volume Monitoring, Ultrasonic Speed Measurement, Air Detector

\section{Introduction}

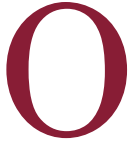

ne of the main goals of hemodialysis is the excess fluid removal in people with renal failure and the achievement of dry weight [1]. On the other hand, the fast reduction of blood water volume due to ultrafiltration via hemodialysis machine can result in clinical complications such as dizziness, nausea, vomiting, hypotension, and muscle cramp.

It should be considered that this situation occurs when the ultrafiltration rate of the machine becomes more than vascular refilling rate in the circulatory system. Under these circumstances, patient's blood water volume gets to a critical threshold and makes adverse conditions for the patient. Therefore, continuous and accurate monitoring of body fluid removal during dialysis is significantly important [2]. This monitoring reduces $\% 77$ of the symptomatic hypotensive episodes [3]. Previously, we proposed some methods using Photoplethysmography and bodyimpedance signals for predicting hypotension episodes in hemodialysis session $[4,5]$.

It is concluded that controlling the blood water volume is specifically important in the dynamic stability of the cardiovascular system [6-8]. To gain desired blood water volume, the relative blood water volume
${ }^{1}$ Ph.D., Associate Professor, Biomedical Engi-

neering Group, Iranian

Research Organization

for Science \& Technol-

ogy, Tehran, Iran

${ }^{2}$ Ph.D., Assistant Profes-

sor, Biomedical Engi-

neering Group, Iranian

Research Organization

for Science \& Technol-

ogy, Tehran, Iran

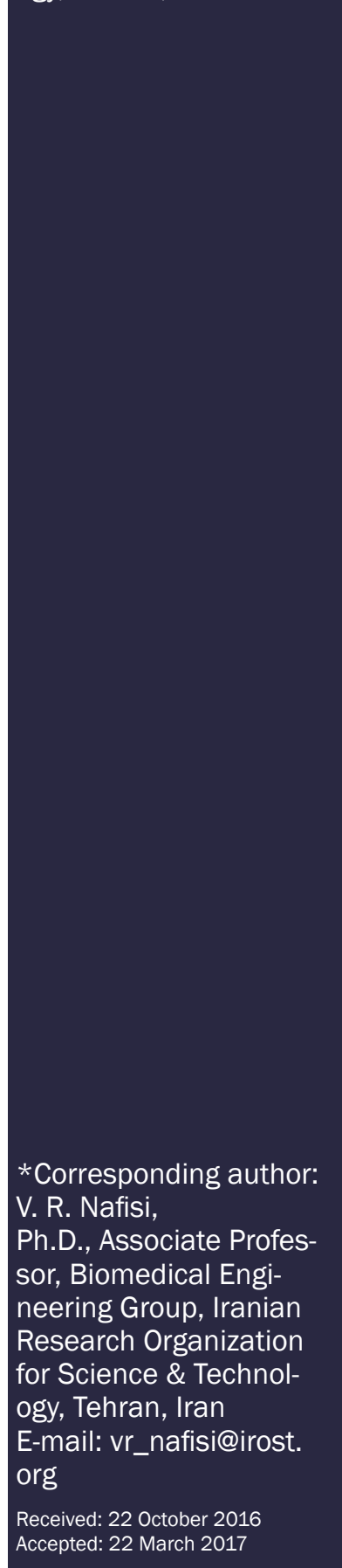


(RBV) is defined. RBV is the ratio of current blood water volume to the initial blood water volume at the start of dialysis.

$$
R B V(t)=\frac{B V(t)}{B V(0)} \times 100 \%
$$

In this formula, $\mathrm{BV}(\mathrm{t})$ and $\mathrm{BV}(0)$ represent blood water volume at time $t$ and at the start of HD, respectively.

The sound speed measurement is used in the blood water volume measurement system of Fresenius hemodialysis machine [9]. In this method, ultrasound waves enter the extracorporeal circuit of hemodialysis machine and the changes in the sound speed in the blood that affect sound transit time are measured. This method assumes that large molecules cannot pass through the dialysis membrane during ultrafiltration; therefore, the more the blood water volume reduces, the more the sound speed in the solution increases, or the more the transit time would reduce. Since the sound speed in the blood depends on its temperature too, the temperature compensation should be considered [10]. Other factors that affect the results of the method are food intake during dialysis, the amount and the timing of the medication intake and body position during dialysis, etc. $[11,12]$. However, using these methods for monitoring hemodialysis machine can reduce the patients' problems $[3,12]$.

In this research, we focused on the "sound" method and tried to improve the measuring method and correspond it with air bubble detector of Iranian hemodialysis machine.

\section{Material and Methods}

\section{Proposed Method Principle}

As mentioned before, the concentration of total blood protein (TPC) changes because of the ultrafiltration during dialysis. The changes of blood water volume due to ultrafiltration and vascular refilling are expressed using the formula (2):

$$
\mathrm{BV}_{\mathrm{ex}}=\mathrm{BV}_{\mathrm{UF}}+\mathrm{BV}_{\mathrm{R}}
$$

Where $\mathrm{BV}_{\mathrm{ex}}$ is the changes in blood water volume in the vascular network, $\mathrm{BV}_{\mathrm{UF}}$ is the ultrafiltrated blood water volume and BVR is the retrieved blood water volume from interstitial space. Also, it can be written as:

$$
\mathrm{BV}(\mathrm{t})=\mathrm{BV}_{\mathrm{ex}}(\mathrm{t})+\mathrm{BV}(0)
$$

On the other hand, it can be written as:

$$
\mathrm{TPC}(\mathrm{t}) \times \mathrm{BV}(\mathrm{t})=\mathrm{TPC}_{\mathrm{ex}} \times \mathrm{BV}_{\mathrm{ex}}(\mathrm{t})+\mathrm{TPC}(0) * \mathrm{BV}(0)
$$

In this formula, TPC represents total protein concentration, and subscripts 0 and t represent the time. The subscript "ex" is related to proteins exchanged between blood and extravascular space that is assumed a constant of about $7 \mathrm{gr} / \mathrm{lit}$ [13].

So, relative changes in blood water volume can be expressed as formula (5):

$$
R B V(t)=\frac{T P C(0)-T P C(t)}{T P C(t)-T P C_{e x}} \times 100
$$

On the other hand, changes in TPC can be determined by measuring the changes of the ultrasonic sound wave speed in the blood [14].

The ultrasound pulse is sent from transducer A to B (Figure 1). The effective speed of sound in the whole track is calculated as [15]:

$$
C_{e}=C+V \cos \theta
$$

where $C_{e}, V$ and $C$ are the effective speed of sound, blood speed and sound speed in the solution, respectively.

The transition time of the ultrasound pulse from $\mathrm{A}$ to $\mathrm{B}$ is equal to:

$$
t_{A B}=\frac{W}{C}+\frac{L}{C+V \cos \theta}
$$

where $L$ and $W$ are the effective path lengths of sound passages in the pipe, through the wall and other components outside the blood path, 


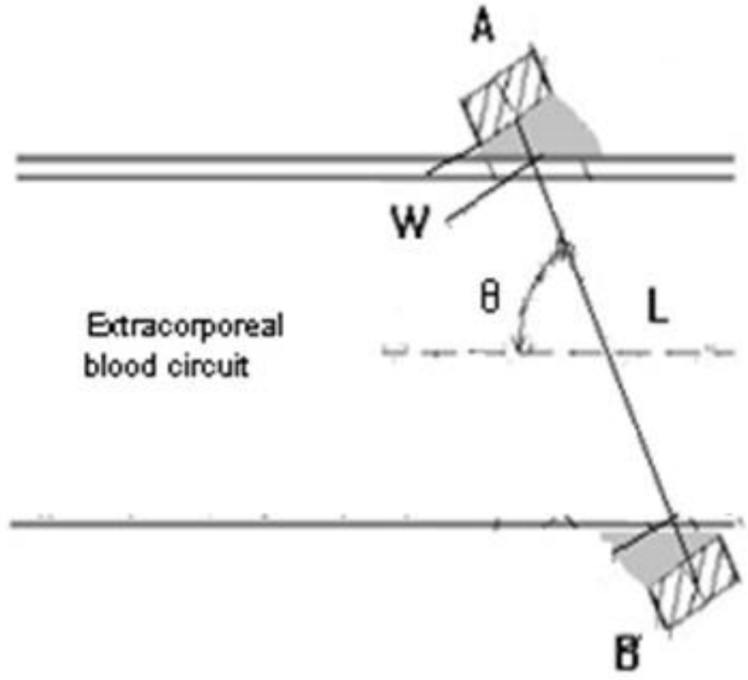

Figure 1: The method of the sound speed calculation in the extracorporeal blood circuit

respectively.

It is obvious that if the voice route in the blood is exactly perpendicular to blood flow,

V $\cos \theta$ will be zero. Therefore, the blood flow speed in the extracorporeal circuit has no effect on the sound speed of the reference. For better eliminating the blood speed effect, the transition time from $\mathrm{B}$ to $\mathrm{A}$ is calculated too:

$$
{ }_{B A}=\frac{W}{C}+\frac{L}{C-V \cos \theta}
$$

Practically, there is $\mathrm{W}<<\mathrm{L}$, so formula (9) is calculated:

$$
C=\frac{L\left(t_{A B}+t_{B A}\right)}{{ }^{2 \times t_{A B} \times{ }_{B A}}}=f(R B V)
$$

Therefore, practically we measure the roundtrip time of sound in the solution and figure out the sound speed in the solution based on this time and the effective diameter of pipe (L).

\section{Sing-around Method}

The sing-around method is one of the ways to increase the accuracy of sound speed mea- surement in the solution [16]. In this method, instead of sending and receiving the ultrasound pulses, a repetition loop is arisen between transmitter and receiver. As the receiver immediately after receiving the ultrasonic pulse stimulates the transmitter again, therefore, an intermittent signal of which period is equal to the sound transit time plus electronic delay between receiver and transmitter, is gained in the receiver.

If the electronic delay of the circuits is completely constant, the sing-around period that is the transit time of the ultrasonic pulse can be measured with high precision. Therefore, measuring the time of several periods and dividing the total time by a number of the periods lead to better results. In our system, to create a sing-around loop in both directions, two control signals that change the receiving and sending roles of transducers, are used [17].

\section{Components of Sing-around Loop} Circuit

The details of the electronic circuits of the system include [17]:

1- The ultrasound transmitter, receiver and the trigger output circuits of the ultrasonic receiver and the loop shift switches. The electronic circuits have been identical as much as possible, and the only difference in each direction of the loop is the difference in the delay of the switches that change the receiving and sending roles of transducers. Accordingly, the electronic delay of 2 states of switches should be low and constant relative to each other, and should not be dependent on temperature conditions and supply voltage. Diode switches have these conditions, while the delay in transistor switches is high and dependent on circumstances.

2- The ring counter that receives the trigger circuit output generates the stop pulse for the timer. The stop pulse is always generated when the ring counter reaches a specified number. As previously noted, the ring counter is used 
for the averaging of the sound transit time in the solution and increasing the accuracy.

3- The timer, which is a high-frequency counter, its input clock is about $108 \mathrm{MHz}$ and measures the time from the beginning of the loop oscillations until the arrival of the stop pulse, giving it to a microcontroller as a number.

The microcontroller and its accessory circuits generate the start pulse of the loop and the changing direction pulse. Therefore, it receives and processes the timing of the timer that is a multi-byte number to calculate and display the sound speed in the fluid sending it to the central computer for further registration and process or for a graphic draw or for monitoring the changes in blood water volume.

At first, the microcontroller changes the loop direction with changing the input, and output switches mode and gives an initial number to the loop counter, then it sends the start pulse to the ultrasound transmitter and timer. The microcontroller or the trigger circuit output can stimulate the ultrasound transmitter. Exactly the same start pulse that stimulates the transmitter resets the timer, which works constantly in the normal situation, and its output is zero to start the timing. After starting the singaround, the ring counter counts the number of ring cycles until it reaches the final number, then it sends the stop pulse to the timer and gives a hardware delay to the microcontroller to read the timer output number.

\section{Transducer Design Parameter}

The ultrasound transmitter and receiver are inserted in a cuvette made of polycarbonate, placed in the extracorporeal circuit [18]. The cuvette is shown in Figure 2. An ultrasound wave is sent to the cuvette, and the gelatinous silicon material is placed between ultrasound transmitter and a cuvette for impedance matching. The pulse transit time, that is dependent on sound speed in the solution, is measured this way.

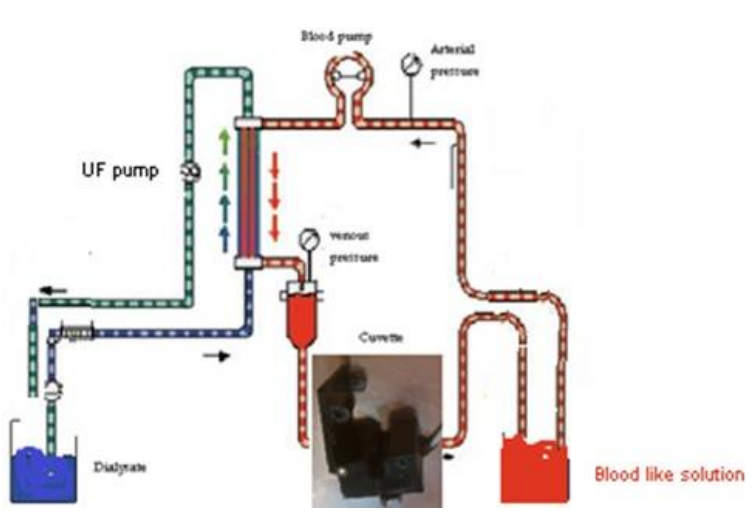

Figure 2: The cuvette used for measuring the sound speed in extracorporeal circuit

Actually, sound speed depends on the blood temperature. In hemodialysis machine, the blood temperature is controlled via the dialysate temperature control.

The transducer should be smaller than the tube diameter; otherwise, the ultrasonic pulse through the tube wall might reach the receiver sooner than the pulse that passes through the fluid.

Moreover, low diameter transducer is not desirable because firstly, its output in the transmitting mode and its received power in the receiving mode would decrease, and secondly, the reduction of the transmitter diameter causes ultrasound output divergence. The working frequency of the transducer with a specified diameter cannot be lower than a certain limit because there is a reduction in its output trigger accuracy in the receiving mode.

On the other hand, high frequencies cause some problems. The higher the frequency, the bigger the near-field in the output ray pattern. Because of the high oscillations of the power in this range, the receiver transducer should be in the far-field of the transmitter transducer [15].

The tube diameter of the extracorporeal circuit is about $4 \mathrm{~mm}$. As a result, in this research, we used the transducer pair with $2.5 \mathrm{MHz}$ fre- 
quency and $3 \mathrm{~mm}$ diameter. This transducer is usually used for detecting the air bubbles in the hemodialysis machine [18].

\section{Results}

For the verification of this method, various laboratory tests have been done; some results were mentioned here. The relation between sound speed and blood concentration, which is related to the hematocrite increase, is gained by using the setup as shown in Figure 2. In this circuit, the blood pump circulates the bloodlike liquid.

The amount of water taken from the blood can be controlled via UF pump setting; therefore, the concentration of the blood water changes.

Whit this circuit, we evaluate the measuring system features. Various factors can cause errors in the results. In sing-around tachometer, because of the mismatches between electronic delays, the total time difference in each direction of the loop is not zero and there is a constant offset. Accordingly, if the number of the loop repetitions increases, the calculated results will have less oscillation and will be more reproducible and accurate (Table 1). The third row of the Table 1 shows that standard deviation of the offset parameter is reduced by increasing the number of the loop repetitions.
This method leads to desired results, and the measurement error reduces by increasing the number of the loop repetitions, although the measuring rate reduces by increasing loop repetitions (Table 2).

In this study, since the change rate of the blood water volume is not high, and the stability of the results is more important, the high loop repetition is preferred.

Laminar or turbulent blood flow is another factor affected by the measuring results. The turbulence can make the standard deviation increase and make noise on the results. Reynolds number is a criterion to define the occurrence probability of the turbulence in the fluid. Reynolds number depends on the viscosity, density and the velocity of the fluid in the pipe and the pipe diameter.

It is shown that laminar flow occurs when Reynolds number $<2000$ [19]. Since the blood flow in the extracorporeal circuit of the hemodialysis machine is not less than $50 \mathrm{ml} /$ min and more than $600 \mathrm{ml} / \mathrm{min}$, it is expected that there is a laminar flow (blood viscosity and density value are about $4 \mathrm{cP}$ and $1060 \mathrm{~kg} /$ $\mathrm{m} 3$, respectively). Figure 3 is drawn from the circuit shown in Figure 2 and determines the measuring error of the sound speed related to the peristaltic pump flow change in the range of $50-600 \mathrm{ml} / \mathrm{min}$.

Table 1: The correlation between time measurement standard deviation and the number of loop repetitions

\begin{tabular}{cccccc} 
The number of loop repetition & $\mathbf{5 0}$ & $\mathbf{1 5 0}$ & $\mathbf{2 5 0}$ & $\mathbf{4 0 0}$ & $\mathbf{6 0 0}$ \\
\hline The average time & 0.16 & 0.147 & 0.16 & 0.155 & 0.158 \\
\hline The time standard deviation & 7 & 5.8 & 6.2 & 2 & 1.8
\end{tabular}

Table 2: The measuring rate and the number of loop repetitions

\begin{tabular}{cccccc} 
The number of loop repetitions & $\mathbf{5 0}$ & $\mathbf{1 5 0}$ & $\mathbf{2 5 0}$ & $\mathbf{4 0 0}$ & $\mathbf{6 0 0}$ \\
\hline The measurement frequency $(\mathrm{Hz})$ & 14.28 & 88.7 & 6.9 & 5.15 & 3.88
\end{tabular}




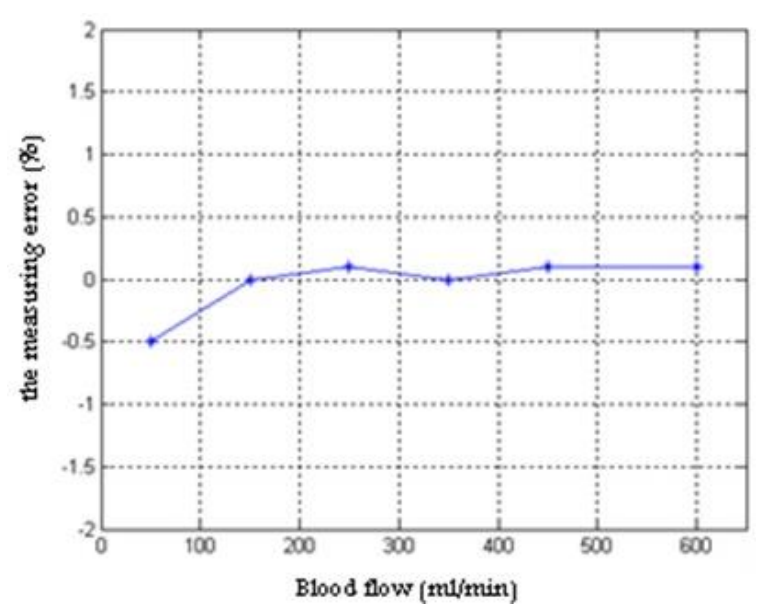

Figure 3: The measuring error related to the blood flow

As it is shown in Figure 3, the measuring error due to the fluid velocity changes is less than $\% 0.5$.

In Figure 4, the estimated sound speed vs. the time from starting of the ultrafiltration is shown. Regarding the fixed adjustment of the ultrafiltration setting during the time, the horizontal axis in Figure 4 represents the amount of fluid condensation. This curve can be used as a calibration diagram.

\section{Discussion}

As it was mentioned, blood volume monitor $(\mathrm{BVM})$ can be used as a controlling sys-

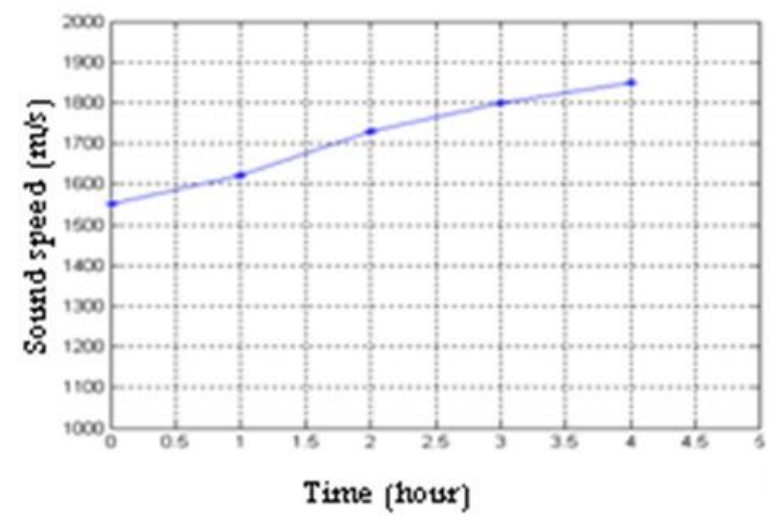

Figure 4: The correlation between the sound speemd and ultrafiltration time tem for estimating each treatment symptom or each patient's activity (such as drinking during dialysis), which can affect the patient's blood water volume. In addition, a simple feedback can be obtained for the control system of the ultrafiltration rate from the blood water volume by BVM [6, 7]. A separate block has been designed for this measurement in some hemodialysis machines [9].

It should be considered that an air bubble detector has to be in the extracorporeal circuit of hemodialysis machine, and it is one of the basic requirements. Nowadays, all hemodialysis machines use ultrasonic detectors for detecting air bubbles. In this study, we evaluated an accurate way for measuring the sound speed to determine the relative blood water volume.

For this purpose, we focused on the bi-directional sing-around method. The bi-directional sing-around method caused the followings:

1 - The measurement accuracy of the transit time increased.

2- The sensitivity to the fluid velocity reduced.

3- The sensitivity to the misalignment of the ultrasonic receiver-transmitter reduced.

A previous study on dialysate flow measurement has shown that the measurement accuracy can be increased 3 or 4 times by this optimized method in combination with the proper selection of electronic circuit components [17].

Regarding the different and specified features of the blood for each patient, Figure 4 is just a typical diagram of the system performance. Practically, two processes should be followed: first, the primary measurement should be done at the beginning of each dialysis session in which the extracorporeal circuit is primed with the $\% 0.9$ venous saline solution, and the measured values should be compared to previously recorded results. Second, by entering the patient's primary blood, the amount corresponding to M0 (in formula 
3 ) is measured and the amount corresponding to $\mathrm{Mt}$ is compared to the primary amount, which is recorded. Then, the critical threshold can be defined and introduced to the machine for each patient separately. For this purpose, the amount corresponding to the relative blood water volume (ratio of $\mathrm{Mt}$ to M0) should be recorded in critical situations (hypotension).

\section{Conclusion}

Ultrasonic waves can be used for measuring the concentration of the soluble proteins in the blood. The purpose of the current study was to expand air bubble detector usage to determine relative blood water volume. The results represent that air bubble detecting and relative blood water volume estimating can be achieved by adding the "sing-around" method to the current air detector device of the hemodialysis machine; air bubble detection is achieved by monitoring the decline in the ultrasonic wave amplitude (because of air bubbles), and the protein concentration measurement is achieved by measuring the transit time of the wave.

The results represent that the designed system has a high potential for achieving the mentioned purposes without adding any additional device to the blood circuit.

The next step is that the equipment is going to be put into practice in the Iranian hemodialysis machine IROST5001.

\section{Acknowledgment}

We thank the members of the designer team of the hemodialysis project. We particularly thank A. Gorgian Mohammadi for the implementation of sing-around circuits.

\section{Conflict of Interest}

None declared.

\section{References}

1. Bosch JP. Hemodialysis: high-efficiency treatments: Churchill Livingstone; 1993.
2. Yu S-J, Kim D-h, Oh D-J, Yu S-H, Kang E-T. Assessment of fluid shifts of body compartments using both bioimpedance analysis and blood volume monitoring. Journal of Korean medical science. 2006;21:75-80. doi.org/10.3346/ jkms.2006.21.1.75.

3. Ramalho V, Malvar B, Pessegueiro P, Amoedo M, Pires C, Aniceto J. Intradialytic blood volume monitoring and its effect on haemodynamic instability. Port J Nephrol Hypert. 2008;22:313-7.

4. Dejake B, Firouzmand M, Nafisi VR. Relative blood volume monitoring based on photometry in hemodialysis process. 2nd Iranian Conference on Advanced Biomedical Engineering in the Cardiovascular field. 2016.

5. Shahabi M, Nafisi VR, Pak F. Prediction of intradiaIytic hypotension using PPG signal features. 22th Iranian Conference on Biomedical Engineering. 2015. doi.org/10.1109//CBME.2015.7404178.

6. Nafisi VR, Eghbal M, Motlagh MR, Yavari F. Fuzzy logic controller for hemodialysis machine based on human body model. J Med Signals Sens. 2011;1:36-48. PubMed PMID: 22606657. PubMed PMCID: 3317763.

7. Azar AT. Biofeedback systems and adaptive control hemodialysis treatment. Saudi J Kidney Dis Transpl. 2008;19:895-903. PubMed PMID: 18974573.

8. Abouie V, Sharifian H, Towhidkhah F, Nafisi VR, Abouie $\mathrm{H}$. Using heart rate and blood concentration data in order to predict hypotension of hemodialysis patients. 19th Iranian Conference on Electrical Engineering (ICEE); 2011.

9. Johner C, Chamney PW, Schneditz D, Krämer M. Evaluation of an ultrasonic blood volume monitor. Nephrology, Dialysis, Transplantation: Official Publication of the European Dialysis and Transplant Association-European Renal Association. 1998;13:2098-103. doi.org/10.1093/ ndt/13.8.2098.

10. Schneditz D, Pogglitsch H, Horina J, Binswanger U. A blood protein monitor for the continuous measurement of blood volume changes during hemodialysis. Kidney international. 1990;38:342-6. doi.org/10.1038/ki.1990.207.

11. Mitra S, Chamney P, Greenwood R, Farrington K. The relationship between systemic and whole-body hematocrit is not constant during ultrafiltration on hemodialysis. J Am Soc Nephrol. 2004;15:463-9. doi.org/10.1097/01.ASN.0000108970.48370.33. 
PubMed PMID: 14747394.

12. Dasselaar JJ, Huisman RM, de Jong PE, Franssen CF. Measurement of relative blood volume changes during haemodialysis: merits and limitations. Nephrology Dialysis Transplantation. 2005;20:2043-9. doi.org/10.1093/ndt/gfi056.

13. Iselin H, Tsinalis D, Brunner FP. Sodium balanceneutral sodium profiling does not improve dialysis tolerance. Swiss Med WKLY. 2001;131:635-9.

14. Schneditz D, Heimel H, Stabinger H. Sound speed, density and total protein concentration of blood. J Clin Chem Clin Biochem. 1989;27:803-6. doi. org/10.1515/cclm.1989.27.10.803. PubMed PMID: 2600553.

15. Christensen D. Ultrasonic bioinstrumentation: Wiley; 1988.
16. Delsing J. A new velocity algorithm for singaround-type flow meters. IEEE Trans Ultrason Ferroelectr Freq Control. 1987;34:431-6. doi. org/10.1109/T-UFFC.1987.26963. PubMed PMID: 18291865.

17. Gorgian-Mohammadi A. Design and manufacture of a precise ultrasonic flowmeter for ultrafiltration measurement during hemodialysis. Science and Technology University, Iran; 2000.

18. Hemodialysis group. Design and manufacture of the hemodialysis machine IROST2000A \& IROST2001D \& IROST4001D reports. Iranian Research Organization for Science \& Technology; 2002-2015.

19. Kinsky R. Applied Fluid Mechanics: McGraw-Hill Book Company; 1992. 